\title{
Concentrating on antennas
}

\author{
The burgeoning field of nanophotonics is taking light into places that did not seem possible. \\ Optical antennas could, in the not-so-distant future, provide a new way of observing the \\ different components of a cell and studying their interaction.
}

Analogy has always been a useful scientific tool. This is particularly true in electromagnetism where, as Maxwell's equations have no intrinsic length scale, a phenomenon commonly used at one wavelength can be scaled to work at any other, provided the appropriate materials are available. One notable example is the concept of the antenna. Antennas have been used to transmit and collect radio waves since the nineteenth century, radically changing our notion of communication in the process. The more recent challenge has been to apply these ideas to electromagnetic radiation at optical frequencies. It is a trend that we have seen before in optics. Both metamaterials and photonic crystals were originally investigated at microwave frequencies; the longer wavelength meant that the structures required were larger and, therefore, easier to fabricate. At their simplest, antennas are one quarter of the wavelength in length. In the optical realm, this means constructing structures that are of the order of hundreds of nanometres: this is not an easy task. But the rapid development of nanotechnology, and the associated fabrication techniques, means that optical antennas are now a reality.

Optical antennas are, thanks to fortuitous timing of several papers that we have accepted, a key theme of the April issue of Nature Photonics, with a total of three primary research papers on the topic, as well as accompanying News and Views articles and a Commentary. This collection highlights the growing importance of these antennas and is recognition of the rapid progress that is being made in the area. Optical antennas can provide the crucial link between two very different size regimes, bridging the gap in scale between the wavelength of visible and infrared light and the dimensions of many of the structures that are of interest in modern

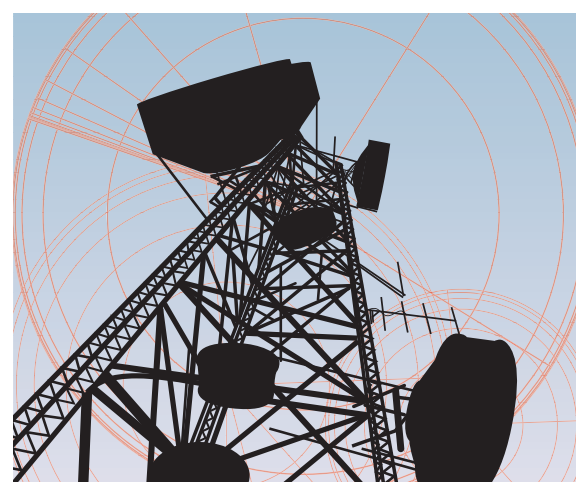

science and technology. The devices that can be realized with today's semiconductor processing are just one example, and it is this that is investigated by Liang Tang and colleagues $^{1}$ from Stanford University on page 226. They construct a photodetector with a germanium active element that has a volume of just $0.00072 \mu \mathrm{m}^{3}$, two orders of magnitude smaller than detectors previously used at this wavelength and far smaller than the limit imposed by the diffraction of light. A gold dipole antenna collects light from a large area and concentrates it into this subwavelength detector, leading to a more sensitive device.

A second example of antennas bridging the scale gap is their use in controlling the emission of light from single atoms and molecules, an idea investigated by Tim Taminiau and co-workers ${ }^{2}$ on page 234. They use a 105-nm-long aluminium antenna at the end of a spatially scanning optical probe to couple light into fluorescent molecules and to collect their emission. The researchers show that in this way an optical antenna can be used to direct emission - a key function of conventional antennas. Rashid Zia from Brown University, writing on page 213 , highlights the potential of such control for the creation of single-photon sources 3 , "Optical antennas have made significant advances towards modifying emission lifetimes and, now, changing the emission pattern."

As with all emerging new technologies, there is a need to expand the functionality of optical antennas. Jörg Merlein and colleagues ${ }^{4}$ show on page 230 how the resonant frequency of an optical antenna can be tuned. They use a different antenna design from the previous two groups, a so-called bow-tie antenna. Two gold triangles, with sides $128 \mathrm{~nm}$ long, are placed pointing at each other. By using an atomic force microscope to vary the gap between the triangles, it is possible to control the wavelength at which the antenna operates.

One field of science that is likely to benefit from these advances is biological imaging. In this month's Commentary ${ }^{5}$ on page 201, Maria Garcia-Parajo discusses the requirements for imaging and sensing applications and the potential of opticalantenna technology. The difficulty is not only the small size of many important molecules, but also the high molecular concentrations at which many of the significant processes we may want to investigate occur. The reduction in detection volume enabled by optical antennas could enable the investigation of processes between individual molecules. It almost goes without saying that further advances are required: the transfer from good idea to universally adopted methodology is never an easy one. However, with such important applications at the end of the road, the motivation is certainly there.

\section{References}

1. Tang, L. et al. Nature Photon. 2, 226-229 (2008).

2. Taminiau, T. H., Stefani, F. D., Segerink, F. B. \& van Hulst, N. F. Nature Photon. 2, 234-237 (2008).

3. Zia, R. Nature Photon. 2, 213-214 (2008).

4. Merlein, J. et al. Nature Photon. 2, 230-233 (2008).

5. Garcia-Parajo, M. F. Nature Photon. 2, 201-203 (2008). 